\title{
SALL4 is a marker of poor prognosis in serous ovarian carcinoma promoting invasion and metastasis
}

\author{
MENGYUAN YANG, XIAOHUI XIE and YILING DING \\ Department of Obstetrics and Gynecology, Second Xiangya Hospital of Central South University, \\ Changsha, Hunan 410011, P.R. China
}

Received October 1, 2015; Accepted December 16, 2015

DOI: $10.3892 /$ or.2016.4545

\begin{abstract}
Spalt-like transcription factor 4 (SALL4) is a transcription factor which is able to regulate development and embryonic stem cell pluripotency, and it is also involved in some tumor progression. However, the role of SALL4 in serous ovarian carcinoma (SOC) is still controversial. In this study, we determined the SALL4 expression in ovarian carcinoma (OC) cell lines and SOC tissues, and explored the clinical value of SALL4 in SOC. The SALL4 mRNA and protein expression in $\mathrm{OC}$ cell lines were examined by real-time PCR and western blotting. The SALL4 protein expression of 91 SOC tissue samples were examined by immunohistochemistry, and the correlation with clinicopathologic features as well as the survivals of SOC patients were analyzed. The invasion potential of OC cells were determined by cytoskeleton immunofluorescence, wound-healing, Transwell and colony formation assays. Our study found SALL4 mRNA and protein were highly expressed in OC cell lines, especially in HO-8910PM. SALL4 protein was highly expressed in SOC tissues, and positively associated with advanced FIGO stage, high histological grade, lymph node involvement, distant metastasis, recurrence and death of SOC (all $\mathrm{P}<0.05)$. Survival analysis showed that high expression of SALL4 indicated poorer overall and progression-free survival $(\mathrm{P}<0.05)$. Univariate and multivariate analysis showed that SALL4 is an independent marker for prognosis prediction $(\mathrm{P}<0.05)$. SALL4 ectopic expression significantly increased the migration, invasion and proliferation capacity of OC cells. In contrast, inhibition of SALL4 markedly decreased these capacities in vitro. In addition, SALL4-interference changed critical signaling involved in cancer progression. In conclusion, SALL4 was highly expressed and correlated with poor
\end{abstract}

Correspondence to: Professor Yiling Ding, Department of Obstetrics and Gynecology, Second Xiangya Hospital of Central South University, 139 Middle Renmin Road, Changsha, Hunan 410011, P.R. China E-mail: dylcsu@sina.cn

Key words: spalt-like transcription factor 4, serous ovarian carcinoma, prognosis, biomarker, invasion, metastasis prognosis in SOC patients, promoting invasion and metastasis of OC cells. SALL4 could be a novel molecular target and prognostic marker for SOC.

\section{Introduction}

Ovarian cancer (OC) is one of the most common causes of postmenopausal female cancer mortality in the world, serous ovarian carcinoma (SOC) accounts for the majority of histological types (1). Although surgery has made great progress in recent years, the overall survival and progression-free survival of SOC are still miserable, largely due to the advanced stage at initial diagnosis in most patients especially in developing countries (2). Patients with advanced SOC [International Federation of Gynecology and Obstetrics (FIGO) stage III/IV] have high metastasis and recurrence rates. In spite of chemotherapy after surgery, which has been the standard of care for almost 40 years worldwide, the response rate is still unsatisfactory because of heterogeneous histopathology. Therefore, there is an urgent need for more sensitive prognotic markers and more effective targets for cancer molecular therapy.

SALL4 is a human homolog of the Drosophila homeotic gene spalt, which plays an important role in embryonic development and self-renewal of embryonic stem cells (3-7). The functional defect of SALL4 may lead to embryonic death or abortion in mouse. There is also research indicated that SALL4 is involved in hematopoiesis and leukemogenesis, the abnormal expression of SALL4 was tightly linked with acute myeloid leukemia $(8,9)$. Recently, a number of studies found SALL4 could take part in invasion and metastasis of various epithelial cancers and correlated with the prognosis, such as gastric cancer, breast cancer, lung cancer, and hepatocellular carcinoma (10-13). Although SOC is also a typical epithelium originated tumor, there is no previous study giving full attention to SALL4 invasion, metastasis and prognosis in SOC. Therefore, the purpose of this study was to explore and assess in detail the role of SALL4 in the progression and prognosis of SOC.

\section{Materials and methods}

Patients and samples. SOC patients who underwent cytoreductive surgery were enrolled in our study. The patients came 
from the Department of Obstetrics and Gynecology, Second Xiangya Hospital of Central South University (Hunan, China) from January 2008 to December 2013. None of the patients received adjuvant chemotherapy before surgery. The diagnosis of SOC for each patient was confirmed by two independent histopathologist. All patients were staged according to the criteria of International Federation of Gynecology and Obstetrics (FIGO) stage system. Clinicopathological parameters of each case including age, serum cancer antigen 125 (CA125), tumor size, histological grade, lymph node involvement, FIGO stage, and recurrence were obtained from patients files. This study was approved by the Research Ethics Committee of the Second Xiangya Hospital of Central South University (China). All patients provided written informed consent for the use of specimens in this study.

Cell culture. OC cell lines A2780,HO-8910PM were purchased from China Center for Type Culture Collection (CCTCC), and SKOV3 cells were obtained from the American Type Culture Collection (ATCC; Manassas, VA, USA). The cell lines were maintained in Dulbecco's modified Eagle's medium (DMEM) or RPMI-1640 medium supplemented with $10 \%$ fetal bovine serum (FBS) at $37^{\circ} \mathrm{C}$ in a humidified atmosphere of $5 \% \mathrm{CO}_{2}$ as recommended by manufacturer's protocol.

Reverse transcriptase-polymerase chain reaction (RT-PCR). TRIzol was used to extract total RNA of the cells, and reverse transcription was conducted with High-Capacity cDNA Synthesis kit (Takara, China). The primer sequences of SALL4 and $\beta$-actin were: SALL4 forward, 5'-AACGCCACTGTCTC CAAGAT-3' and reverse, 5'-AAGCAGCATAGCAACAA TCG-3'; and $\beta$-actin forward, 5'-CTGGGACGACATGGA GAAAA-3' and reverse, 5'-AAGGAAGGCTGGAAGAG TGC-3'.

PCR product $(10 \mu \mathrm{l})$ was obtained for agarose gel electrophoresis, and image was captured by gel imaging system. Ratio between the target gene and the corresponding $\beta$-actin of each sample was determined, respectively. Semi-quantitative measurement was performed for the electrophoretic bands, and the mRNA expression was recorded.

Quantitative real-time PCR. Total RNA was extracted using Qiagen RNeasy Mini kit (Qiagen, Valencia, CA, USA) following manufacturer's instructions. Reverse transcription was carried out using High-Capacity Reverse Transcription kit with RNase inhibitor (Applied Biosystems, Foster City, CA, USA). Quantitative PCR was performed using SYBR Green Real-time PCR Master Mix (Toyobo, Osaka, Japan). Amplification was done with Abio-7100 (Qiagen) using the following parameters: $95^{\circ} \mathrm{C}(10 \mathrm{~min}), 40$ cycles of $95^{\circ} \mathrm{C}(15 \mathrm{sec})$, $60^{\circ} \mathrm{C}(60 \mathrm{sec})$, and $72^{\circ} \mathrm{C}(20 \mathrm{sec})$. All measurements were performed in duplicate. The following primers were used: SALL4 forward, 5'-GCGAGCTTTTACCACCAAAG-3' and reverse, 5'-CACAACAGGGTCCACATTCA-3'; and $\beta$-actin forward, 5'-AGCGAGCATCCCCCAAAGTT-3' and reverse, 5'-GGGCACGAAGGCTCATCATT-3'.

Vector construction and transfection. The SALL4 ectopic expressions and knockdown lentivirus as well as their control lentivirus were all purchased from GeneChem (Shanghai,
China). The SALL4 knockdown target sequences were: shSALL4-1, GTACCTAAAGACAGAGACA; shSALL4-2, CCACCAAAGGCAACTTAAA; and shSALL4-3, GGAACC AGTACACCAGCAT.

Western blot analysis. Total cell lysates were harvested in NP-40 lysis buffer (150 mM NaCl, 1\% Nonidet P-40, $50 \mathrm{mM}$ Tris, $\mathrm{pH} 8.0$, protease inhibitor cocktail) and protein concentrations were determined by BCA protein assay (Bio-Rad Laboratories, Inc., Hercules, CA, USA). Equal amounts of proteins from each lysate were resolved by SDS-PAGE and then transferred to PVDF membranes. Membranes were blocked with buffer containing 5\% skim milk and $0.1 \%$ Tween-20 in PBS for $1 \mathrm{~h}$ at room temperature with gentle shaking. Primary antibodies (SALL4 and $\beta$-actin) were incubated overnight at $4^{\circ} \mathrm{C}$ with gentle shaking, followed by secondary antibody incubation at room temperature for $1 \mathrm{~h}$ with gentle shaking. The following antibodies were used: SALL4 (\#sc-101147) and $\beta$-actin (\#sc-47778) (both from Santa Cruz Biotechnology, Santa Cruz, CA, USA), NF-кB (\#16502; Abcam, Cambridge, MA, USA), $\beta$-catenin (\#sc-65480; Santa Cruz Biotechnology), ERK (\#36911), and p-ERK (\#4819) (both from Abcam).

Immunohistochemistry (IHC) and immunofluorescence. The tissue specimens were fixed in $10 \%$ formalin and embedded in paraffin. Sections $(4 \mathrm{~mm})$ were cut and placed on silane-coated slides for immunohistochemical studies. Part of the specimens was stained with H\&E and microscopically examined to confirm the diagnosis. The paraffin sections were stained for SALL4 (\#sc-101147, 1:150 dilution; Santa Cruz Biotechnology) using streptavidin-peroxidase system (Zhongshan Goldenbridge Biotechnology, Beijing, China) according to manufacturer's protocol. Appropriate positive and negative controls were included for each run of IHC. Immunostaining was evaluated by two independent pathologists simultaneously on a multi-headed microscope without knowledge of the patient information. Only nuclear staining was considered positive for SALL4. SALL4 expression was scored according to the percentage of tumor cells stained positive for SALL4, with 0 denoting $<5 \%$ of tumor cells stained positive, $1,5-30 \%$ of tumor cells stained positive, $2,31-50 \%$ of tumor cells stained positive, $3,51-80 \%$ of tumor cells stained positive, and $4,>80 \%$ of tumor cells stained positive. The low expression was defined as $0-1$, the high expression was defined as 2-4 (12).

Cells were plated on glass coverslips. After $24 \mathrm{~h}$, the cells were rinsed with PBS and fixed with $4 \%$ paraformaldehyde for $15 \mathrm{~min}$, and cell membrane was permeabilized using $0.25 \%$ Triton X-100 for $10 \mathrm{~min}$. The cells were washed in PBS three times for $5 \mathrm{~min}$. Then, the cells were incubated with $1 \%$ BSA in PBST for 30 min to block unspecific binding of the antibodies, and then incubated with $100 \mathrm{nM}$ rhodamine phalloidin (\#PHDR1; Cytoskeleton, Inc., Denver, CO, USA) at room temperature in the dark for $30 \mathrm{~min}$. After three washes, the nucleus was counterstained with $100 \mathrm{nM}$ DAPI (4'6-diamidino-2-phenylindole) for $30 \mathrm{sec}$.

Wound-healing, Transwell and colony formation assay. For the wound-healing assay, cells were seeded into 35-mm dishes. When cells reached $90 \%$ confluence, a scratch was made with 


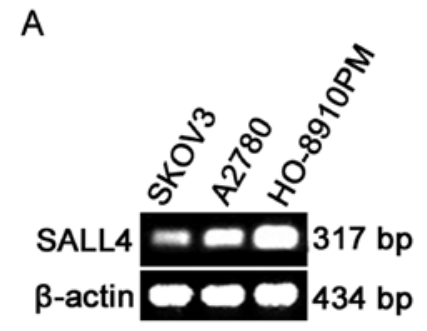

C

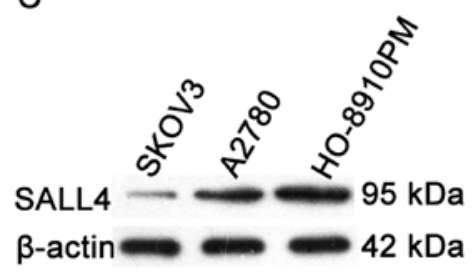

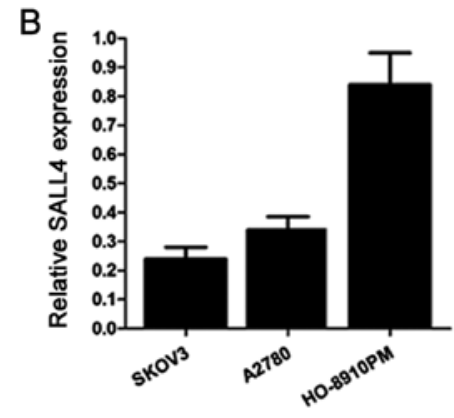

D

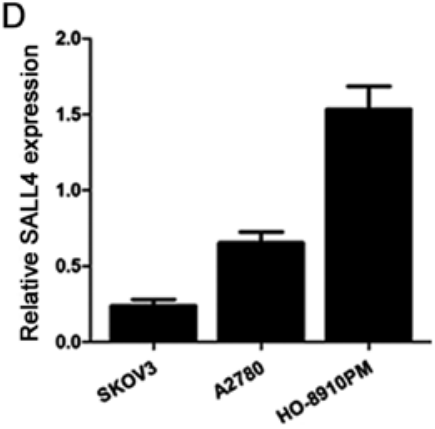

Figure 1. Spalt-like transcription factor 4 (SALL4) is overexpressed in ovarian carcinoma (OC) cells. (A) The SALL4 mRNA expression was detected by semiquantitative RT-PCR indicating that SALL4 was expressed in OC cells, and the HO-8910PM cells had the highest, while SKOV3 had the lowest expression. (B) Real-time PCR results showed similar SALL4 mRNA expression to semi-quantitative RT-PCR. (C) The representative western blot results showed that SALL4 protein was expressed in OC cells. (D) The western blotting band density was measured by ImageJ software, it also showed HO-8910PM cells had the highest while SKOV3 had the lowest SALL4 protein expression.

a 10- $\mu$ l pipette tip. The cells were then cultured for another $24 \mathrm{~h}$ and micrographs were taken. For the Transwell assay, approximately $1 \times 10^{5}$ cells were placed into the upper chamber of the insert with Matrigel (BD Biosciences, Bedford, MA, USA). The cells were cultured in serum-free medium in $5 \%$ $\mathrm{CO}_{2}$ for $12-24 \mathrm{~h}$, the cells in the upper chamber were removed and the membrane was stained with a solution containing $0.1 \%$ crystal violet and $20 \%$ methanol. The number of cells in the lower membrane of the inserts was counted. Each experiment was performed in triplicate, and 3 random fields were chosen for cell number quantification. For colony formation assay, $1 \times 10^{3}$ cells were seeded into 6 -well plates (Corning, Inc., Corning, NY, USA) and cultured for 2 weeks at $37^{\circ} \mathrm{C}$ and $5 \% \mathrm{CO}_{2}$. The number of colonies per dish was counted after staining with crystal violet, only colonies larger than $40 \mu \mathrm{m}$ were taken into account. All the experiments were conducted with 3 replicates.

Multipathway reporter array. The Cignal Finder 10-Pathway Reporter Array (SABiosciences) was used to detect signaling changed by SALL4 interfered. Cells were plated in 96-well plates at a density of 30,000 cells/well and allowed to attach to the plate for $24 \mathrm{~h}$. Next, the cells were lysed, and relative firefly luciferase activity was calculated and normalized to the constitutively expressed Renilla luciferase.

Patients follow-up. The follow-up period was from January 2008 to 31 December, 2014. Follow-up of all surviving patients included serum CA125 levels, abdomen ultrasonography every 4 months, especially during the first 2 years. For suspected disease progression, computed tomography (CT) or magnetic resonance imaging (MRI) were performed. The follow-up period was defined as the interval from the date of operation to the date of death or last follow-up. We censored survival at 5 years after the initial surgery. Deaths from other causes were treated as censored cases. The primary outcome was overall survival (OS), defined as the interval between surgery and death regardless of etiology, and secondary end points were progression-free survival (PFS) defined by the interval between surgery and tumor progression.

Statistical analysis. All data were analyzed using the statistical software SPSS, version 17.0 for Windows (SPSS, Chicago, IL, USA). The Fisher's exact test was used for statistical analysis of categorical data, whereas independent t-tests were used for continuous data. Survival curves were constructed using the Kaplan-Meier method and evaluated using the log-rank test. The Cox proportional hazards regression model was established to identify factors which were independently associated with the OS and PFS of OC patients. P-value $<0.05$ was considered statistically significant.

\section{Results}

Aberrant expression of SALL4 $m R N A$ and protein in OC cell lines. We detected the SALL4 mRNA expression in OC cell lines of SKOV3, HO-8910PM and A2780 by RT-PCR and real-time PCR. The RT-PCR results showed that SALL4 mRNA was expressed in all OC cell lines, and the expression level was the highest in HO-8910PM, and the lowest in SKOV3 cells (Fig. 1A). The high expression level of SALL4 in OC cell lines was also confirmed by real-time PCR, and the expression of SALL4 in HO-8910PM was 3.5-fold higher than SKOV3 (Fig. 1B). Moreover, we performed western 
Table I. Correlation between SALL4 expression and clinicopathological variables of serous ovarian cancer patients.

\begin{tabular}{llll}
\hline & \multicolumn{2}{c}{ SALL4 expression } & \\
\cline { 2 - 3 } Variables & Low (38) & High (53) & P-value \\
\hline
\end{tabular}

Age (years)

$\begin{array}{llll}\leq 50 & 25 & 32 & \\ >50 & 13 & 21 & 0.664\end{array}$

Performance status

$\begin{array}{lrrr}\text { ECOG } 0 & 36 & 50 & \\ \text { ECOG }>0 & 2 & 3 & 0.655 \\ \text { FIGO stage } & & & \\ \text { Early (I/II) } & 22 & 4 & \\ \text { Advanced (III/IV) } & 16 & 49 & \mathbf{< 0 . 0 0 1} \\ \text { CA125 (U/ml) } & & & \\ \leq 34 & 16 & 17 & \\ >34 & 22 & 36 & 0.380\end{array}$

Histological grade

$\begin{array}{llll}\text { Low } & 19 & 10 & \\ \text { High } & 19 & 43 & \mathbf{0 . 0 0 3}\end{array}$

Residual disease

after surgery (mm)

$\begin{array}{llll}\leq 10 & 24 & 23 & \\ >10 & 14 & 30 & 0.089\end{array}$

LN involvement

$\begin{array}{llll}\text { Absent } & 28 & 20 & \\ \text { Present } & 10 & 33 & \mathbf{0 . 0 0 1}\end{array}$

Distant metastasis

$\begin{array}{lrrr}\text { Absent } & 36 & 24 & \\ \text { Present } & 2 & 29 & <\mathbf{0 . 0 0 1} \\ \text { Recurrence } & & & \\ \text { Absent } & 20 & 15 & \\ \text { Present } & 18 & 38 & \mathbf{0 . 0 2 8}\end{array}$

End status

$\begin{array}{lccc}\text { Alive } & 29 & 22 & \\ \text { Dead } & 9 & 31 & \mathbf{0 . 0 0 1}\end{array}$

Significant results $(\mathrm{P}<0.05)$ are given in bold. ECOG, Eastern Cooperative Oncology Group; FIGO, International Federation of Gynecology and Obstetrics; LN, lymph node. SALL4 low expression, IHC score 0 to 1 ; SALL4 high expression, IHC score 2 to 3.

blot analysis to detect the SALL4 protein expression in OC cell lines, the results also showed the SALL4 expression could be detected in all OC cell lines, and the expression level is also the highest in HO-8910PM, and the lowest in SKOV3 (Fig. 1C and D). These results concluded that SALL4 mRNA and protein were aberrantly expressed in OC cell lines.

Aberrant expression of SALL4 in SOC tissues by IHC. We then explored the SALL4 expression in SOC patients. We collected 91 cases of paraffin-embedded SOC specimens and examined the SALL4 protein expression by IHC. The positive expression was defined as granular brown substance which located mainly in the nucleus. The protein expression pattern was categorized into two groups: high expression (IHC level 2 to 4 ) versus low expression (IHC level 0 to 1). According to the results, 53 samples (58.2\%) showed high expression and 38 samples $(41.8 \%)$ exhibited low SALL4 staining in the tumor (Table I). We also collected 10 cases of normal ovarian tissues samples which were removed from non-tumor diseases, and the SALL4 staining for these samples were all negative. Representative samples of SALL4 expression in the tumor and ovarian tissues are shown in (Fig. 2). These results indicate that SALL4 was upregulated in SOC tissues, but absent in normal ovarian tissues.

SALL4 expression is correlated with clinicopathological characteristics of SOC patients. The relation of SALL4 protein expression and the clinicopathological characteristics of SOC patient after surgery were further analyzed. Results showed that SALL4 high expression was significantly positively correlates with the important clinicopathological features of that advanced FIGO stage, high histological grade, lymph node involvement, distant metastasis, recurrence and death (all $\mathrm{P}<0.05)$. However, SALL4 expression had no significance relation with age, performance status, serum CA125 level and residual disease (all $\mathrm{P}>0.05$ ) (Table I). These results indicated that SALL4 expression may be correlated with the survival of SOC after surgery.

SALL4 expression is correlated with poor survival of SOC patients. We then explored whether the expression of SALL4 correlated with the survival of SOC patients after surgery. Kaplan-Meier survival analysis revealed that the 1-, 3- and 5-year overall survival (OS) rates of high SALL4 expression group patients were $77.2,49.4$ and $31.5 \%$, respectively, which were significantly poorer than the low expression group of 97.3, 75.4 and 62.1\% ( $\mathrm{P}<0.05)$ (Fig. 3A). Correspondingly, the 1-, 3- and 5-year progression survival (PFS) rates of high SALL4 expression group patients were 67.0, 27.9 and 13.4\%, respectively, which were also obviously poorer than the low expression group of 83.0, 64.9 and $42.2 \%(\mathrm{P}<0.05)$ (Fig. 3B). Because the grade of SOC is important for the prognosis of SOC according to recent research, we also explored the survival of SALL4 expression in low- and high-grade group, respectively. It showed that high SALL4 expression SOC patients had shorter OS and PFS than that of low SALL4 expression in both low-grade and high-grade subgroups (Fig. 3C-E, $\mathrm{P}<0.05$, respectively). These results indicated that SALL4 high expression was tightly correlated with poor survival of SOC patients and may have potential as a prognostic marker.

SALL4 expression is an independent risk factor for survival of SOC patients. To assess the role of SALL4 expression for prognosis prediction of SOC patients after surgery, we performed the univariate and multivariate Cox proportional hazards regression model. The univariate survival analysis revealed that performance status ECOG $>0$, advanced FIGO stage, high histological grade, residual disease $>10 \mathrm{~mm}$, lymph node involvement, distant metastasis and SALL4 expression were risk factors for the overall survival of SOC patients. However, the multivariate analysis indicated that 


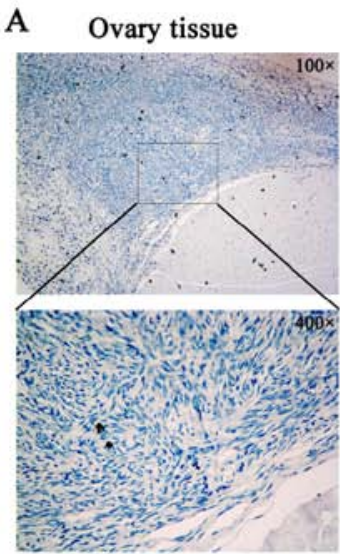

$(-)$

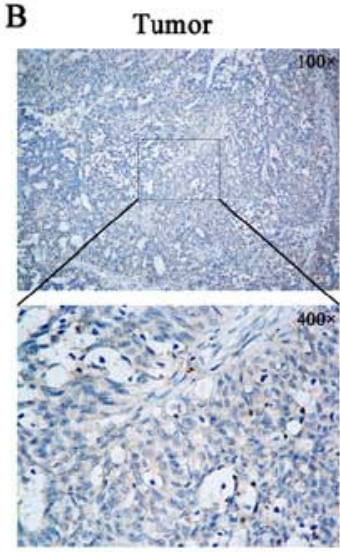

$(-)$

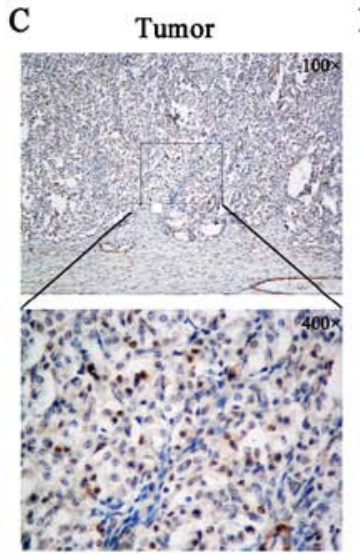

$(+)$

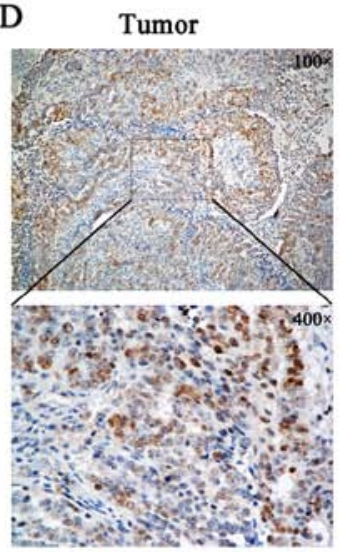

$(++)$

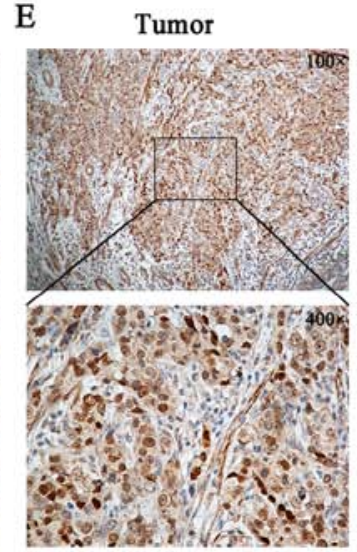

$(+++)$

Figure 2. SALL4 expression levels in ovarian tissue and serous OC (SOC) tissues detected by immunohistochemistry. (A) SALL4 showed negative expression in ovarian tissue. (B) Negative expression of SALL4 in SOC tissue. (C) Weakly positive expression of SALL4 in SOC tissue. (D) Moderately positive expression of SALL4 in SOC tissue. (E) Strongly positive expression of SALL4 in SOC tissue. The original magnification of images on the top set: x100, and on the lower set: $x 100$.
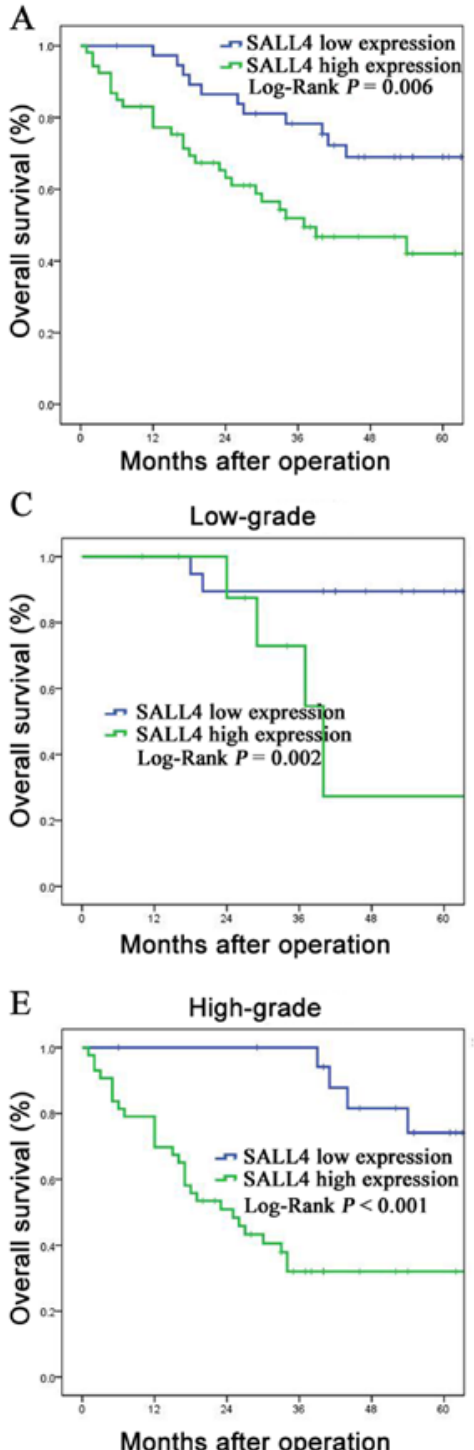

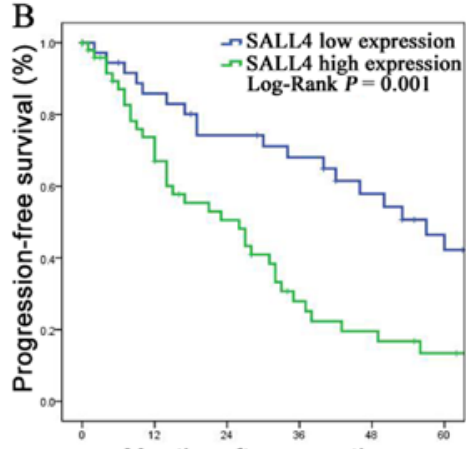

Months after operation
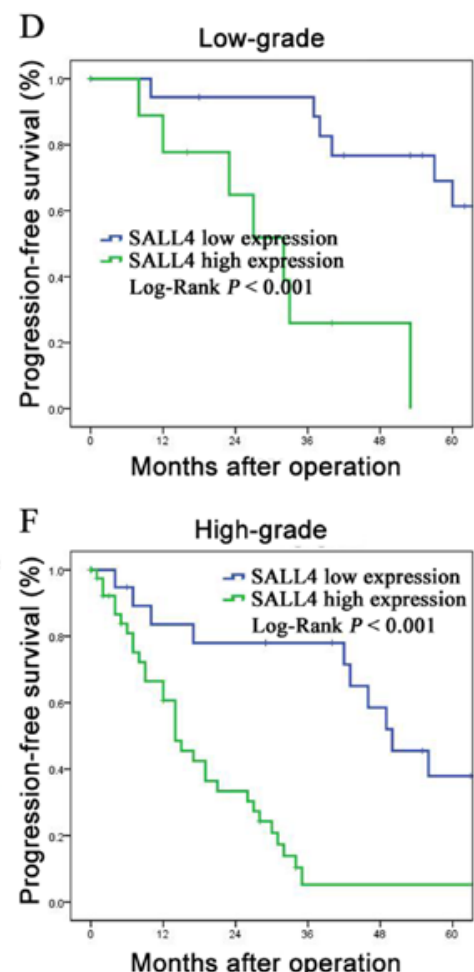

Figure 3. Overall survival and progression-free survival of SOC patients were analyzed according to SALL4 expression. (A) Survival curves indicate that high SALL4 expression SOC patients had poorer overall survival (OS) than low expression group ( $\mathrm{P}=0.006)$. (B) Survival curves show that high SALL4 expression SOC patients had poorer progression-free survival (PFS) than low expression group $(\mathrm{P}=0.001)$. (C) Survival curves indicate that in low-grade group high SALL4 expression SOC patients had shorter OS and PFS (D) than low-grade group ( $\mathrm{P}=0.002, \mathrm{P}<0.001$, respectively). (E) Survival curves indicate that in high-grade group high SALL4 expression SOC patients had shorter OS and PFS (F) than low-grade group (both $\mathrm{P}<0.001$ ). 
Table II. Univariate and multivariate analysis of clinicopathological variables and SALL4 protein expression for OS of serous ovarian cancer patients.

\begin{tabular}{|c|c|c|c|c|c|}
\hline \multirow[b]{2}{*}{ Variables } & \multirow[b]{2}{*}{$\mathrm{n}$} & \multicolumn{2}{|c|}{ Univariate analysis } & \multicolumn{2}{|c|}{ Multivariate analysis } \\
\hline & & HR $(95 \%$ CI $)$ & P-value & HR $(95 \% \mathrm{CI})$ & P-value \\
\hline \multicolumn{6}{|l|}{ Age (years) } \\
\hline$<50$ & 57 & 1 & & & \\
\hline$\geq 50$ & 34 & $0.845(0.441-1.619)$ & 0.612 & NA & \\
\hline \multicolumn{6}{|l|}{ Performance status } \\
\hline ECOG 0 & 86 & 1 & & 1 & \\
\hline $\mathrm{ECOG}>0$ & 5 & $2.869(1.921-4.930)$ & $<0.001$ & $2.380(1.366-3.581)$ & 0.002 \\
\hline \multicolumn{6}{|l|}{ FIGO stage } \\
\hline Early (I/II) & 26 & 1 & & 1 & \\
\hline Advanced (III/IV) & 65 & $3.146(1.292-6.733)$ & $<0.001$ & $2.214(1.236-4.004)$ & 0.015 \\
\hline \multicolumn{6}{|l|}{ CA125 (U/ml) } \\
\hline$\leq 34$ & 33 & 1 & & & \\
\hline$>34$ & 58 & $1.223(0.637-2.349)$ & 0.546 & NA & \\
\hline \multicolumn{6}{|l|}{ Histological grade } \\
\hline Low & 29 & 1 & & 1 & \\
\hline High & 62 & $2.075(1.355-6.979)$ & 0.007 & $1.468(0.261-2.564)$ & 0.332 \\
\hline \multicolumn{6}{|c|}{ Residual disease after surgery (mm) } \\
\hline$\leq 10$ & 47 & 1 & & 1 & \\
\hline$>10$ & 44 & 4.667 (2.337-9.322) & $<0.001$ & $2.991(1.232-7.260)$ & 0.015 \\
\hline \multicolumn{6}{|l|}{ LN involvement } \\
\hline Absent & 48 & 1 & & 1 & \\
\hline Present & 43 & $3.407(1.739-6.678)$ & 0.002 & $0.776(0.338-1.782)$ & 0.550 \\
\hline \multicolumn{6}{|l|}{ Distant metastasis } \\
\hline Absent & 60 & 1 & & 1 & \\
\hline Present & 31 & $4.199(1.849-8.454)$ & $<0.001$ & $3.312(1.704-6.562)$ & 0.004 \\
\hline \multicolumn{6}{|l|}{ SALL4 expression } \\
\hline Negative & 38 & 1 & & 1 & \\
\hline Positive & 53 & $2.545(1.713-5.524)$ & $<0.001$ & $2.188(1.242-4.379)$ & 0.003 \\
\hline
\end{tabular}

Significant results $(\mathrm{P}<0.05)$ are given in bold. OS, overall survival; HR, hazard risk ratio; CI, confidence interval; NA, not analysis.

only performance status ECOG $>0$, advanced FIGO stage, residual disease $>10 \mathrm{~mm}$, distant metastasis and SALL4 expression were significant independent prognostic factors for OS (Table II). Moreover, analysis for PFS revealed that advanced FIGO stage, CA125 >34 U/ml, high histological grade, residual disease $>10 \mathrm{~mm}$, lymph node involvement, distant metastasis and SALL4 expression were significant risk factors in the univariate survival analysis, but only advanced FIGO stage, lymph node involvement, distant metastasis and SALL4 expression were independent prognosis factors for PFS in multivariate survival analysis (Table III). These results indicated that high SALL4 expression was an independent risk factor for OS and PFS of SOC patients after surgery.

SALL4 expression promotes invasion and proliferation of $O C$ cells. To further study the role of SALL4 in OC, we established SALL4 stable ectopic expression SKOV3 cells (named SKOV3-SALL4) and SALL4 knockdown HO-8910PM cells (named HO-8910PM-shSALL4), as well as their control cells. The expression of SALL4 in these cells was verified by real-time PCR and western blot analysis, and we chose HO-8910PM-shSALL4-3 for the next experiments (Fig. 4A-a1-2 and B-b1-2). The migration, invasion and metastasis capacity of SALL4-mediated OC cells was confirmed by cytoskeleton immunofluorescence, woundhealing and Transwell assays. The cytoskeleton showed SKOV3-SALL4 cells had extending shape compared with control cells, and SALL4 knockdown cells exhibited inverse morphology change (Fig. 5A-a1-2). Wound-healing assay revealed that SKOV3-SALL4 cells had significantly faster closure of the wound area than their control cells (Fig. 5B-b1). Transwell assay also showed that more SKOV3-SALL4 cells penetrated the Matrigel compared to control 
Table III. Univariate and multivariate analysis of clinicopathological variables and SALL4 protein expression for PFS of serous ovarian cancer patients.

\begin{tabular}{|c|c|c|c|c|c|}
\hline \multirow[b]{2}{*}{ Variables } & \multirow[b]{2}{*}{$\mathrm{n}$} & \multicolumn{2}{|c|}{ Univariate analysis } & \multicolumn{2}{|c|}{ Multivariate analysis } \\
\hline & & $\mathrm{HR}(95 \% \mathrm{CI})$ & P-value & $\mathrm{HR}(95 \% \mathrm{CI})$ & P-value \\
\hline \multicolumn{6}{|l|}{ Age (years) } \\
\hline$<50$ & 57 & 1 & & & \\
\hline$\geq 50$ & 34 & $0.868(0.509-1.479)$ & 0.602 & NA & \\
\hline \multicolumn{6}{|l|}{ Performance status } \\
\hline ECOG 0 & 86 & 1 & & 1 & \\
\hline $\mathrm{ECOG}>0$ & 5 & $1.700(0.891-3.210)$ & 0.071 & $1.536(0.421-2.830)$ & 0.138 \\
\hline \multicolumn{6}{|l|}{ FIGO stage } \\
\hline Early (I/II) & 26 & 1 & & 1 & \\
\hline Advanced (III/IV) & 65 & $3.628(1.903-6.034)$ & $<0.001$ & $2.860(1.425-5.661)$ & $<0.001$ \\
\hline \multicolumn{6}{|l|}{ CA125 (U/ml) } \\
\hline$\leq 34$ & 33 & 1 & & 1 & \\
\hline$>34$ & 58 & $1.968(1.109-3.493)$ & 0.021 & $1.257(0.638-2.475)$ & 0.508 \\
\hline \multicolumn{6}{|l|}{ Histological grade } \\
\hline Low & 29 & 1 & & 1 & \\
\hline High & 62 & $2.123(1.660-3.878)$ & 0.008 & $1.012(0.451-2.269)$ & 0.977 \\
\hline \multicolumn{6}{|c|}{ Residual disease after surgery (mm) } \\
\hline$\leq 10$ & 47 & 1 & & 1 & \\
\hline$>10$ & 44 & $2.940(1.719-5.030)$ & $<0.001$ & $1.541(0.831-2.855)$ & 0.170 \\
\hline \multicolumn{6}{|l|}{ LN involvement } \\
\hline Absent & 48 & 1 & & 1 & \\
\hline Present & 43 & $4.878(2.765-8.608)$ & $<0.001$ & $2.785(1.349-5.748)$ & 0.006 \\
\hline \multicolumn{6}{|l|}{ Distant metastasis } \\
\hline Absent & 60 & 1 & & 1 & \\
\hline Present & 31 & $4.461(2.822-7.566)$ & $<0.001$ & $3.080(1.819-5.278)$ & 0.003 \\
\hline \multicolumn{6}{|l|}{ SALL4 expression } \\
\hline Negative & 38 & 1 & & 1 & \\
\hline Positive & 53 & $3.269(1.263-5.042)$ & $<0.001$ & $2.961(1.614-4.721)$ & 0.007 \\
\hline
\end{tabular}

Significant results $(\mathrm{P}<0.05)$ are given in bold. $\mathrm{PFS}$, progression-free survival.

A

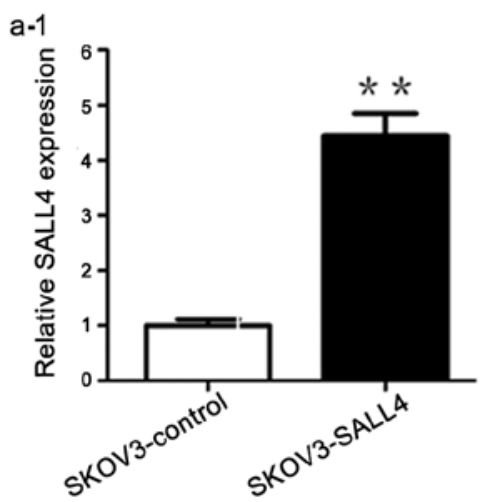

a-2

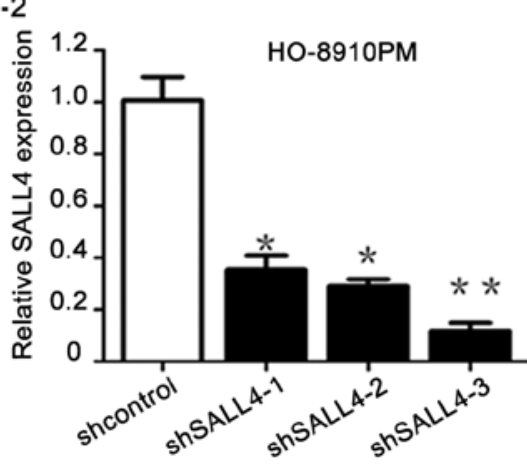

B

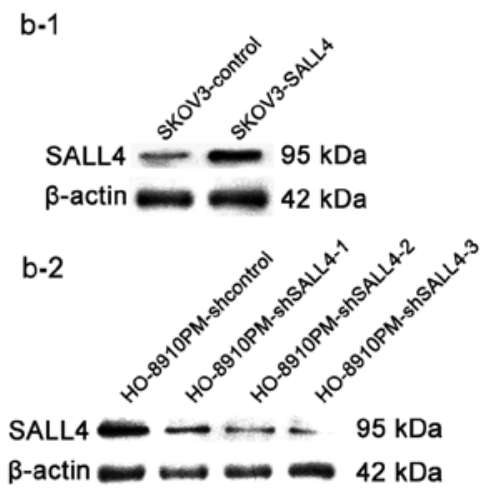

Figure 4. Validation of SALL4 expression in SALL4-interfered OC cells. (A-a1) SALL4 mRNA expression levels were significantly upregulated in SKOV3-SALL4 cells compared to control cells. (A-a2) SALL4 mRNA expression levels were significantly downregulated in HO-8910PM-shSALL4 cells cpmpared to control cells, and HO-8910PM-shSALL4-3 showed the greatest inhibition. (B-b1 and b2) SALL4 protein expression levels were consistent with mRNA detected by western blot analysis. 


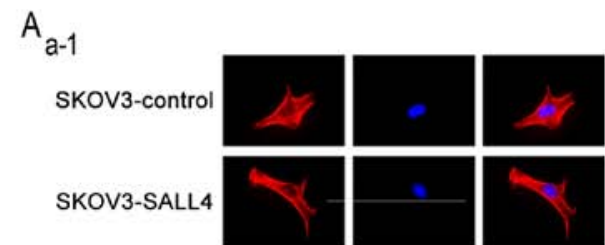

$B$

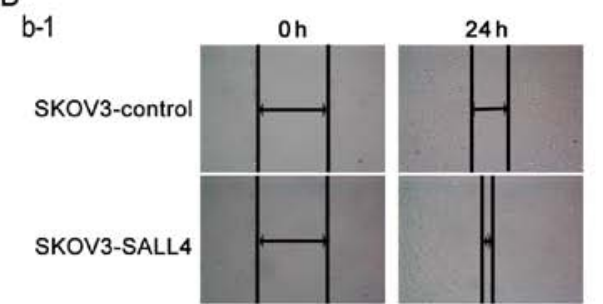

C

c-1
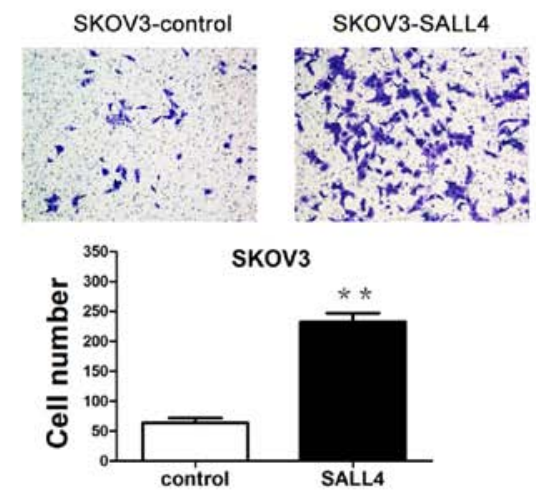

$D_{d-1}$

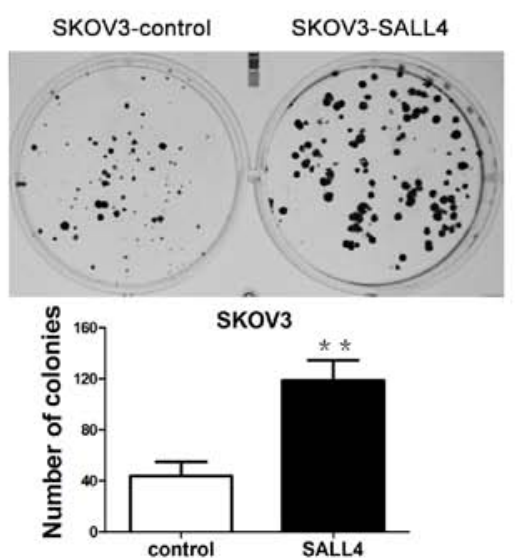

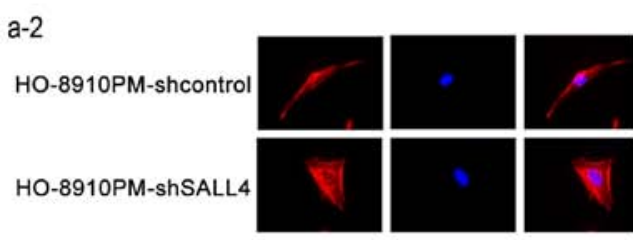

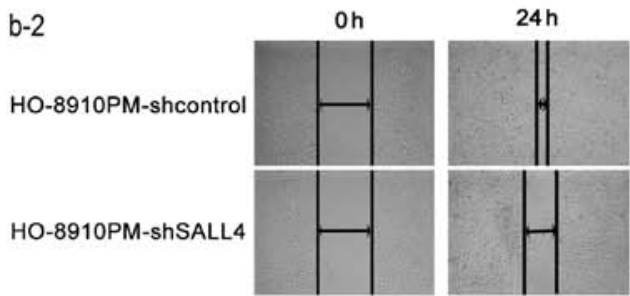

c-2
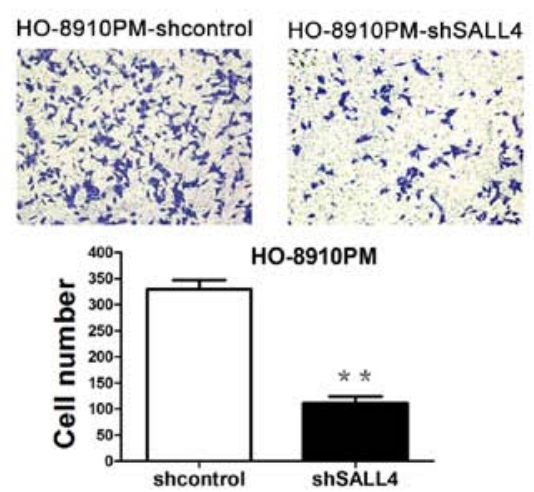

d-2

HO-8910PM-shcontrol HO-8910PM-shSALL4
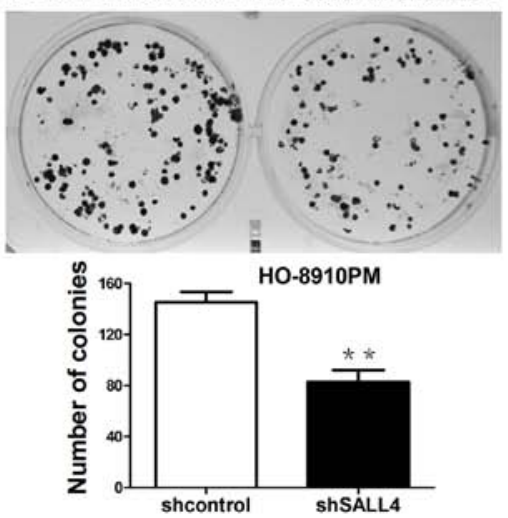

Figure 5. SALL4 promotes migration, invasion and proliferation of OC cells. (A-a1) Immunofluorescence (IF) for cytoskeleton of SKOV3-SALL4, showed SALL4 ectopic expression SKOV3 cells with an extending shape. (A-a2) IF showed SALL4 knockdown HO-8910PM cells were shrunken. (B-b1) Woundhealing assay showed SALL4 ectopic expression increased the closure of wound area of SKOV3. (B-b2) Wound-healing assay showed SALL4 knockdown decreased the closure of wound area of HO-8910PM. (C-c1) Tranwell assay showed SALL4 ectopic expression increased the invasion cell number of SKOV3. (C-c1) SALL4 knockdown decreased the invasion of HO-8910PM cells. (D-d1) SALL4 ectopic expression increased the colonies of SKOV3 cells. (D-d2) SALL4 knockdown decreased the colonies of HO-8910PM cells. ${ }^{*} \mathrm{P}<0.05 ;^{* *} \mathrm{P}<0.01$.

cells (Fig. 5C-c1). In contrast, SALL4 knockdown markedly reduced the migratory and invasive capacity of HO-8910PM cells (Fig. 5B-b2 and C-c2). Consistent with these results, SALL4 ectopic expression promoted colony formation, and SALL4 knockdown had the opposite effect (Fig. 5D-d1-2). These results concluded that SALL4 promoted the invasion and metastasis potential of OC cells.

SALL4 expression activates signaling associated with cancer progression. To explore the potential mechanism of SALL4 regulated in SOC, we performed multiple signal reporter analysis. It showed MAPK/ERK, NF- $\kappa \mathrm{B}$ and WNT signaling were activated in SKOV3-SALL4 cells comparing with the control cells. While knockdown of SALL4 in HO-8910PM cells had the opposite results (Fig. 6A and C). Next, we used western blot analysis to detect the change of the key member of these three pathways, and found $\mathrm{p}-\mathrm{ERK}, \mathrm{NF}-\kappa \mathrm{B}$ and $\beta$-catenin were upregulated in SKOV3-SALL4 cells, while downregulated in HO-8910PM-shSALL4 cells comparing with their control cells (Fig. 6B and D). These results indicated that SALL4 
A
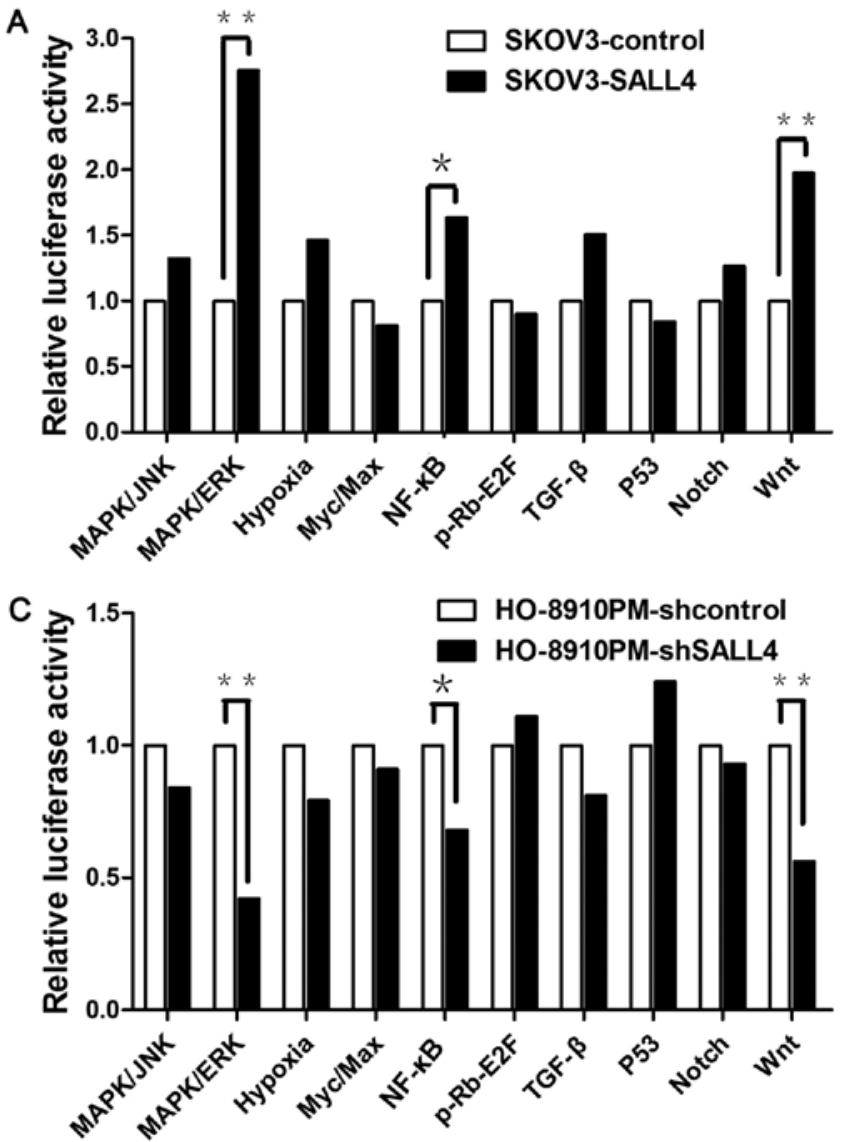

B

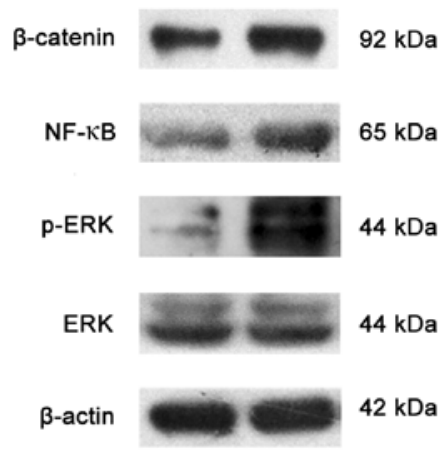

D

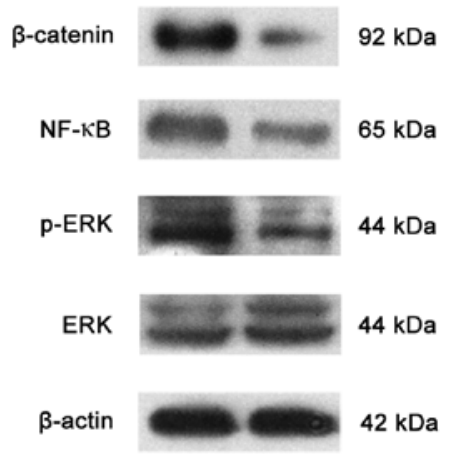

Figure 6. Signaling changes in SALL4-interfered OC cells. (A) Multiple signal reporter showed MAPK/ERK, NF-кB and WNT signaling activation were upregulated in SKOV3-SALL4 cells. (B) Western blot analysis showed p-ERK, NF- $\kappa B$ and $\beta$-catenin were upregulated in SKOV3-SALL4 cells. (C) MAPK/ ERK, NF- $\kappa B$ and WNT signaling activation were downregulated in HO-8910-shSALL4 cells. (D) p-ERK, NF- $\mathrm{B}$ and $\beta$-catenin were downregulated in HO-8910-shSALL4 cells.

interference indeed changed the pivotal signaling associated with cancer progression.

\section{Discussion}

SOC patients are common in clinic, and many of the patients are at advanced stages when diagnosed $(1,14)$. SOC is different to other solid tumors, it can directly broadly invade adjacent organs or peritoneal cavity via peritoneal fluid. Because of the diffuse nature of SOC, the objective of surgery is often cytoreductive, but not radical $(15,16)$. Therefore, the chemotherapy and molecular targeted therapies gradually play a more important role in the postoperative treatment of SOC. Although $70 \%$ of SOC patients initially experience a response to chemotherapy or molecular biotherapy, they eventually die of the disease relapse, and the probability of the disease progression is hard to predict (17), indicating that the existing therapeutic molecular targets and prognosis markers are not sensitive and efficacious enough. Therefore, there is an urgent need to better understand the mechanisms involved in SOC invasion and progression, and development of more effective therapeutic molecular targets and prognostic biomarkers.

SALL4 is a zinc finger transcription factor of SALL gene family, and is the human homolog of the Drosophila homeotic gene spalt, located on human chromosome 20.q13.2 $(6,18)$.
SALL4 used to be considered as an important transcription factor involved in various normal organs and tissue development. The mutation or abnormal expression of SALL4 correlated with Okihiro/Duane-Radial Ray syndrome, Ivic Syndrome, ventricular septal defect and premature ovarian failure $(6,18,19)$. There are also studies that found SALL4 is important for the maintenance of embryonic stem cell pluripotency and self-renewal properties $(3,5,7)$. These characteristics of SALL4 in development and embryonic stem cells are similar to many oncogenes. Therefore, many recent studies correlated SALL4 to neoplasms. For example, some studies found SALL4 was overexpressed in various types of human hematopoietic malignancies such as acute myelocytic and lymphocytic leukemia $(8,9,20)$. Studies also revealed SALL4 was aberrantly expressed in solid tumors such as lung cancer, breast cancer, hepatocellular carcinoma and gastric cancer and SALL4 high expression predicted adverse progression and poor outcome $(11-13,21)$. The role of SALL4 with reproductive system cancers is still controversial. Previous studies showed that SALL4 was important for spermatogonial progenitor cells (SPC) differentiation, and SALL4 was considered as a diagnostic marker for germ cell tumors $(20,22,23)$. However, SALL4 in non-germ cell tumors such as ovary cancer is obscure. It was reported that SALL4 was detected in $20 \%$ of cases or 
more of serous carcinoma of the ovary (24). Another study found SALL4 expression in human ovary tissue and epithelial OC OVCAR-3 cells, and emerging results suggested that acquisition of invasiveness in OC cells was accompanied by the undifferentiated phenotype $(25,26)$. These controversial phenomena triggered our great interest to explore and clarify the role of SALL4 in SOC.

Our study revealed that SALL4 mRNA and protein were expressed in OC cell lines, IHC assay also indicated SALL4 was aberrant highly expressied in SOC tissues (53/91, 58.2\%). These results were different to a previous study where SALL4positive cases were $29 \%$ (23/78) (24). We analyzed the reasons and found that in the previous study there was no clinicopathological information of cases, nor was FIGO stage or histological grade provided, so a sampling error is possible. Our study included specimens with detailed clinicopathological features and follow-up information. Further analysis of SALL4 expression with the clinicopathological factors uncovered that high SALL4 expression was significantly positively correlated with adverse clinicopathological factors, this was also similar to the study in other solid tumors. Survival analysis showed that SOC patients with high SALL4 expression had poorer OS and PFS, indicated SALL4 might be a cancer-promoting gene and therapeutic molecular target in SOC. Univariate and multivariate analysis both showed that high SALL4 expression was the main influential factor for OS and PFS of SOC patients, indicated that SALL4 was a novel prognosis biomarker for SOC. Furthermore, our cytology experiments confirmed SALL4 expression was crucial to maintain the invasion and proliferation capacities of OC cells, knockdown of SALL4 significantly decreased the invasion and proliferation of OC cells. These results were comparable to previous studies on the function of SALL4 in solid tumors, and further verified SALL4 was important for SOC progression (10-12,20,27). How SALL4 prompts SOC invasion and metastasis need further study. In a study on leukemia, it was found that SALL4 could bind to $\beta$-catenin and synergistically enhanced the $\mathrm{Wnt} / \beta$-catenin signaling pathway, this was confirmed by another study that SALL4 had a TCF/LEF-binding site and could be activated by LEF1 and TCF4E $(6,8,25)$. There were also studies reporting SALL4 involved in HOXA9, c-Myc and PTEN-AKT pathway, these genes all potentially correlate with epithelial-mesenchymal transition (EMT) $(9,12,27)$. This study indicated that MAPK/ERK, NF- $\kappa \mathrm{B}$ and WNT signaling were activated with SALL4 ectopic expression, and downregulated by SALL4 knockdown. These pathways are all closely correlated with EMT (28). The functional role of SALL4 in development and embryonic stem cell differentiation was also association with EMT (29,30). EMT plays an important role in promoting SOC invasion and metastasis (3,31-33). Therefore, we hypothesized that SALL4 could facilitate SOC progression via EMT, but this needs further study.

Collectively, our results suggested that SALL4 was significantly highly expressed in OC cell lines and SOC tissues. SALL4 high expression was correlated with adverse tumor progression and could be a novel molecular target and prognostic marker for SOC. SALL4 was crucial for the invasion and metastasis of OC cells. However, the detailed molecular biological mechanisms of how SALL4 promotes SOC progression and poor survival need further in vitro and in vivo study.

\section{References}

1. Jayson GC, Kohn EC, Kitchener HC and Ledermann JA: Ovarian cancer. Lancet 384: 1376-1388, 2014.

2. Cannistra SA: Cancer of the ovary. N Engl J Med 351: 2519-2529, 2004.

3. Xiong J: SALL4: Engine of cell stemness. Curr Gene Ther 14: 400-411, 2014

4. Tan MH, Au KF, Leong DE, Foygel K, Wong WH and Yao MW: An Oct4-Sall4-Nanog network controls developmental progression in the pre-implantation mouse embryo. Mol Syst Biol 9: 632, 2013.

5. Rao S, Zhen S, Roumiantsev S, McDonald LT, Yuan GC and Orkin SH: Differential roles of Sall4 isoforms in embryonic stem cell pluripotency. Mol Cell Biol 30: 5364-5380, 2010.

6. Uez N, Lickert H, Kohlhase J, de Angelis MH, Kühn R, Wurst W and Floss T: Sall4 isoforms act during proximal-distal and anterior-posterior axis formation in the mouse embryo. Genesis 46: 463-477, 2008.

7. Zhang J, Tam WL, Tong GQ, Wu Q, Chan HY, Soh BS, Lou Y, Yang J, Ma Y, Chai L, et al: Sall4 modulates embryonic stem cell pluripotency and early embryonic development by the transcriptional regulation of Pou5f1. Nat Cell Biol 8: 1114-1123, 2006.

8. Ma Y, Cui W, Yang J, Qu J, Di C, Amin HM, Lai R, Ritz J, Krause DS and Chai L: SALL4, a novel oncogene, is constitutively expressed in human acute myeloid leukemia (AML) and induces AML in transgenic mice. Blood 108: 2726-2735, 2006.

9. Li A, Yang Y, Gao C, Lu J, Jeong HW, Liu BH, Tang P, Yao X, Neuberg D, Huang G, et al: A SALL4/MLL/HOXA9 pathway in murine and human myeloid leukemogenesis. J Clin Invest 123: 4195-4207, 2013.

10. Ardalan Khales S, Abbaszadegan MR, Abdollahi A, Raeisossadati R, Tousi MF and Forghanifard MM: SALL4 as a new biomarker for early colorectal cancers. J Cancer Res Clin Oncol 141: 229-235, 2015 .

11. Zhang L, Xu Z, Xu X, Zhang B, Wu H, Wang M, Zhang X, Yang T, Cai J, Yan Y, et al: SALL4, a novel marker for human gastric carcinogenesis and metastasis. Oncogene 33: 5491-5500, 2014.

12. Masuda S, Suzuki K and Izpisua Belmonte JC: Oncofetal gene SALL4 in aggressive hepatocellular carcinoma. N Engl J Med 369: 1171, 2013.

13. Kobayashi D, Kuribayshi K, Tanaka M and Watanabe N: SALL4 is essential for cancer cell proliferation and is overexpressed at early clinical stages in breast cancer. Int J Oncol 38: 933-939, 2011.

14. Jelovac D and Armstrong DK: Recent progress in the diagnosis and treatment of ovarian cancer. CA Cancer J Clin 61: 183-203, 2011.

15. Yang D, Sun Y, Hu L, Zheng H, Ji P, Pecot CV, Zhao Y, Reynolds S, Cheng H, Rupaimoole R, et al: Integrated analyses identify a master microRNA regulatory network for the mesenchymal subtype in serous ovarian cancer. Cancer Cell 23: 186-199, 2013.

16. Bast RC Jr, Hennessy B and Mills GB: The biology of ovarian cancer: New opportunities for translation. Nat Rev Cancer 9: 415-428, 2009

17. Jemal A, Tiwari RC, Murray T, Ghafoor A, Samuels A, Ward E, Feuer EJ and Thun MJ; American Cancer Society: Cancer statistics, 2004. CA Cancer J Clin 54: 8-29, 2004.

18. Kohlhase J, Schubert L, Liebers M, Rauch A, Becker K, Mohammed SN, Newbury-Ecob R and Reardon W: Mutations at the SALL4 locus on chromosome 20 result in a range of clinically overlapping phenotypes, including Okihiro syndrome, Holt-Oram syndrome, acro-renal-ocular syndrome, and patients previously reported to represent thalidomide embryopathy. J Med Genet 40: 473-478, 2003.

19. Al-Baradie R, Yamada K, St Hilaire C, Chan WM, Andrews C, McIntosh N, Nakano M, Martonyi EJ, Raymond WR, Okumura S, et al: Duane radial ray syndrome (Okihiro syndrome) maps to $20 \mathrm{q} 13$ and results from mutations in SALL4, a new member of the SAL family. Am J Hum Genet 71: 1195-1199, 2002.

20. Zhang X, Yuan X, Zhu W, Qian H and Xu W: SALL4: An emerging cancer biomarker and target. Cancer Lett 357: 55-62, 2015.

21. Kobayashi D, Kuribayashi K, Tanaka M and Watanabe N: Overexpression of SALL4 in lung cancer and its importance in cell proliferation. Oncol Rep 26: 965-970, 2011. 
22. Hobbs RM, Fagoonee S, Papa A, Webster K, Altruda F Nishinakamura R, Chai L and Pandolfi PP: Functional antagonism between Sall4 and Plzf defines germline progenitors. Cell Stem Cell 10: 284-298, 2012.

23. Trinh DT, Shibata K, Hirosawa T, Umezu T, Mizuno M, Kajiyama $\mathrm{H}$ and Kikkawa F: Diagnostic utility of CD117, CD133, SALL4, OCT4, TCL1 and glypican-3 in malignant germ cell tumors of the ovary. J Obstet Gynaecol Res 38: 841-848, 2012

24. Miettinen M, Wang Z, McCue PA, Sarlomo-Rikala M, Rys J, Biernat W, Lasota J and Lee YS: SALL4 expression in germ cell and non-germ cell tumors: A systematic immunohistochemical study of 3215 cases. Am J Surg Pathol 38: 410-420, 2014.

25. Böhm J, Sustmann C, Wilhelm C and Kohlhase J: SALL4 is directly activated by TCF/LEF in the canonical Wnt signaling pathway. Biochem Biophys Res Commun 348: 898-907, 2006.

26. Cao L, Shao M, Schilder J, Guise T, Mohammad KS and Matei D: Tissue transglutaminase links TGF- $\beta$, epithelial to mesenchymal transition and a stem cell phenotype in ovarian cancer. Oncogene 31: 2521-2534, 2012.

27. Li A, Jiao Y, Yong KJ, Wang F, Gao C, Yan B, Srivastava S, Lim GS, Tang P, Yang H, et al: SALL4 is a new target in endometrial cancer. Oncogene 34: 63-72, 2015.

28. Sánchez-Tilló E, Liu Y, de Barrios O, Siles L, Fanlo L, Cuatrecasas M, Darling DS, Dean DC, Castells A and Postigo A EMT-activating transcription factors in cancer: Beyond EMT and tumor invasiveness. Cell Mol Life Sci 69: 3429-3456, 2012.
29. Polyak K and Weinberg RA: Transitions between epithelial and mesenchymal states: Acquisition of malignant and stem cell traits. Nat Rev Cancer 9: 265-273, 2009.

30. Lim J and Thiery JP: Epithelial-mesenchymal transitions: Insights from development. Development 139: 3471-3486, 2012.

31. Yi BR, Kim TH, Kim YS and Choi KC: Alteration of epithelial-mesenchymal transition markers in human normal ovaries and neoplastic ovarian cancers. Int J Oncol 46: 272-280, 2015.

32. Chao TK, Yo YT, Liao YP, Wang YC, Su PH, Huang TS and Lai HC: LIM-homeobox transcription factor 1, alpha (LMX1A) inhibits tumourigenesis, epithelial-mesenchymal transition and stem-like properties of epithelial ovarian cancer. Gynecol Oncol 128: 475-482, 2013.

33. Davidson B, Holth A, Hellesylt E, Tan TZ, Huang RY, Tropé C, Nesland JM and Thiery JP: The clinical role of epithelial-mesenchymal transition and stem cell markers in advanced-stage ovarian serous carcinoma effusions. Hum Pathol 46: 1-8, 2015. 